\title{
Combining morphological analysis and Bayesian networks for strategic decision support
}

\author{
A de Waal|k T Ritchey
}

Received: 27 September 2006; Revised: 23 April 2007; Accepted: 24 April 2007

\begin{abstract}
Morphological analysis (MA) and Bayesian networks (BN) are two closely related modelling methods, each of which has its advantages and disadvantages for strategic decision support modelling. MA is a method for defining, linking and evaluating problem spaces. BNs are graphical models which consist of a qualitative and quantitative part. The qualitative part is a cause-and-effect, or causal graph. The quantitative part depicts the strength of the causal relationships between variables. Combining $\mathrm{MA}$ and $\mathrm{BN}$, as two phases in a modelling process, allows us to gain the benefits of both of these methods. The strength of MA lies in defining, linking and internally evaluating the parameters of problem spaces and BN modelling allows for the definition and quantification of causal relationships between variables. Short summaries of MA and BN are provided in this paper, followed by discussions how these two computer aided methods may be combined to better facilitate modelling procedures. A simple example is presented, concerning a recent application in the field of environmental decision support.
\end{abstract}

Key words: Morphological analysis, Bayesian networks, strategic decision support.

\section{Introduction}

Strategic decision support often involves developing scenarios and creating complex strategy models. This presents us with a number of difficult methodological problems. Firstly, many of the factors involved are non-quantifiable, since they contain strong socio-political dimensions and conscious self-reference among actors. Furthermore, the uncertainties inherent in such problem complexes are in principle irreducible and often cannot be described or delineated fully. This means that traditional quantitative methods, such as causal modelling and simulation, are relatively useless.

Added to this, the creative process involved in such studies is often difficult to trace. One seldom has an adequate "audit trail" describing the iterative process from problem

\footnotetext{
${ }^{*}$ Corresponding author: Meraka Institute, CSIR, PO Box 395, Pretoria, 0001, South Africa, email: adewaal@csir.co.za.

${ }^{\dagger}$ Ritchey Consulting, Pajalagatan 56, SE-162 65, Vllingby, Sweden.
} 
formulation, through alternative generation to specific solutions or conclusions. Without some form of traceability, there is little possibility of scientific control over results, let alone reproducibility.

There are, however, methods and techniques that provide structure and guidance for thinking systematically about complex strategic decisions. Employed properly, these result in dynamic, interactive inference ("what-if") models that support complex decision making. Importantly, one of the implicit outcomes of such a modelling process is a shared set of concepts among experts, and a better understanding of the wider context.

Although there is no concise, unanimously agreed upon general definition of a (scientific) model, we feel that the following criteria meet what most analysts and researchers would consider a minimal definition (Compare with Doty \& Glick, 1994).

- A model must contain two or more constructs that can serve as variables, i.e. dimensions which can support a range of states or values.

- One must be able to establish relationships (causal, statistical, logical, etc.) between these states or values.

- These relationships must be falsifiable (if the model applies to the empirical world).

Two corollaries to these criteria are:

- Inputs can be given, and outputs obtained.

- Hypotheses can be formulated.

This is a very general definition of the concept of "model" which, we feel, suffices to illuminate the general modelling processes described below.

Morphological Analysis (MA) and Bayesian networks (BN) are two closely related modelling methods that can be employed systematically for strategic decision support. Each has its advantages and disadvantages for modelling complex processes and systems. MA allows small groups of subject specialists to define, link and internally evaluate the parameters of complex problem spaces easily, thus creating a solution space and flexible inference model. However, MA cannot easily treat hierarchal structure and causal relationships.

BN modelling allows for such causal and hierarchal relationships, but is more difficult to employ in the initial problem formulation phase of the modelling process. Combining MA and $\mathrm{BN}$, as two phases in the modelling process, allows one to gain the benefits of both of these modelling methods.

This paper contains a short presentation of Morphological Analysis and Bayesian networks as strategic decision support modelling methods. A case study involving the use of both these methods - in sequence - is also described. The study concerns the development of a prototype instrument to assess the environmental impact of different fire-fighting methods under different conditions. 


\section{Morphological analysis}

Morphological Analysis (MA) was developed by Zwicky $(1967,1969)$ - the Swiss-American astrophysicist and aerospace scientist - as a general method for structuring and investigating the total set of relationships contained in multi-dimensional, usually non-quantifiable, problem complexes.

Zwicky applied this method to such diverse fields as astrophysics, the development of propulsive power plants and propellants, and the legal aspects of space travel and colonisation. He founded the Society for Morphological Research and enthusiastically advanced the "morphological approach" for some 40 years — from the early 1930's until his death in 1974.

More recently, morphological analysis has been extended and applied by a number of analysts and researchers in the USA and Europe in the field of policy analysis and futures studies (Rhyne 1981, 1995a, 1995b; Coyle, 1995, 1996). In 1996, one of the authors (Ritchey) developed computer support for general morphological analysis. Since then, researchers at FOI (the Swedish National Defence Research Agency) have carried out some fifty projects employing this method (Ritchey 1997, 1998, 2002, 2003; Eriksson \& Ritchey, 2002; Ritchey \& Stenström, 2002; Stenström \& Westrin, 2004).

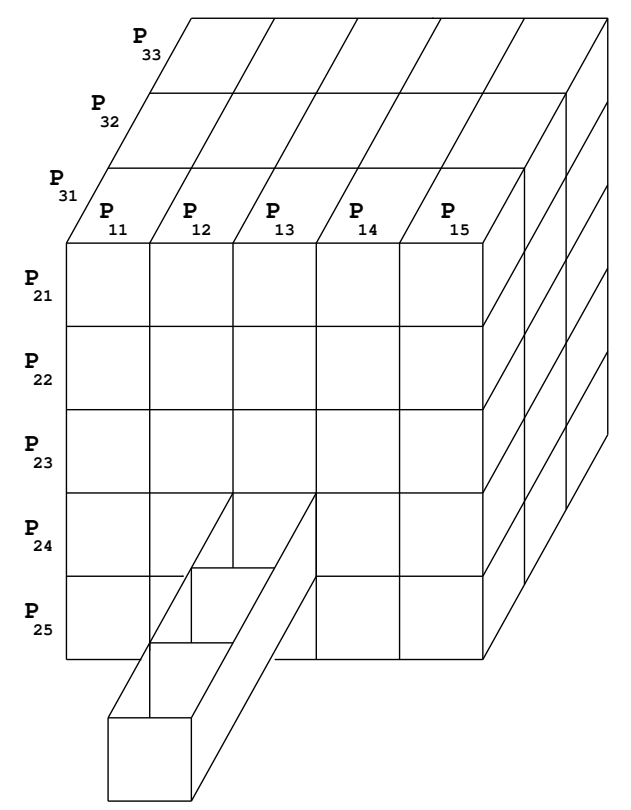

Figure 1: A 3-parameter Zwicky box containing 75 cells or 'configurations' (Zwicky, 1969, p. 118).

MA begins by identifying and defining the dimensions (which eventually become the variables) of the problem complex to be investigated. Thereafter, each variable is assigned a range of relevant values or conditions. A morphological box - also fittingly known as a "Zwicky box" - is constructed by setting the variables against each other in an $n$-dimensional matrix, essentially a variable space (see Figure 1). Each cell of the $n$ dimensional box contains one particular value or condition from each of the variables, and 
thus marks out a particular state or configuration of the problem complex as a whole.

For example, imagine a simple problem complex which we define as consisting of three variables - let us say "colour", "texture" and "size." In order to conform to Figure 1, let us further define the first two variables as consisting of 5 discrete "values" or conditions each $(e . g$. colour $=$ red, green, blue, yellow, brown and texture $=$ smooth, rough, bumpy, patterned, slick) and the third consisting of 3 values (size = large, medium, small) . We then have $5 \times 5 \times 3=75$ cells in the Zwicky box, each containing 3 conditions - i.e. one from each variable (e.g. red, rough, large). The entire 3-dimensional matrix is, in Zwicky's terms, a morphological field containing all of the (formally) possible relationships involved.

The point is to examine all of the configurations in the field, in order to establish which of them are possible, viable, practical, interesting, etc., and which are not. In doing this, we mark out in the field what might be called a 'solution space'. The 'solution space' of a Zwickian morphological field consists of the subset of configurations which satisfy some criteria.

Naturally, if one is working with more than three variables — which is certainly the case in any but the simplest of problems - then they cannot be represented in 3-dimensional space. For this reason, MA employs an alternative method of representing the multiple variables of the problem complex, the so-called morphological field. The field below (Figure 22) represents the 75-configuration Zwicky-box in Figure 1. The marked configuration in Figure 2 corresponds to one cell in the Zwicky-box.

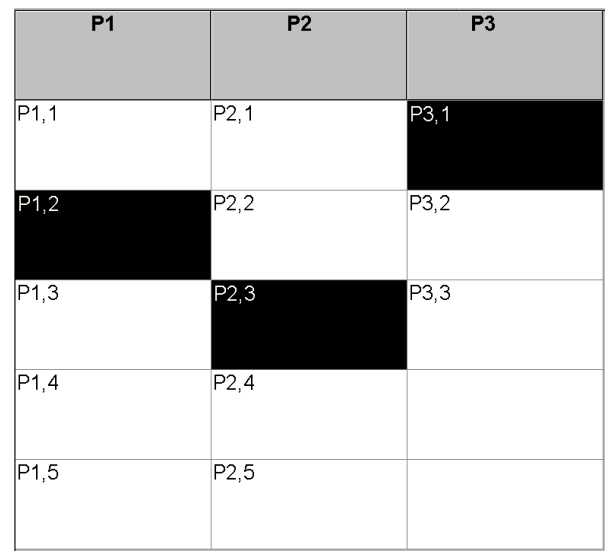

Figure 2: A three parameter morphological field containing 75 possible configurations, one selected.

Morphological models typically contain 8-10 variables and tens of thousands to millions of possible (formal) configurations, far too many to examine individually. Thus, one must be able to reduce the number of configurations in a field, so that only those that meet certain criteria remain. The main criterion is that a configuration is internally consistent, i.e. that is does not contain conditions that are mutually contradictory. In fact, there are usually many such contradictory pairs of conditions in a typical morphological field. To the extent that a particular pair of conditions is considered to be a contradiction, all those configurations containing this pair of conditions would also be internally inconsistent. 
Fields are reduced by comparing each condition of each variable with every other condition of every other variable, and asking the question: Can these two conditions coexist? This is done by doing a cross-consistency assessment (CCA) with the help of a cross-consistency matrix (Figure 3, below). This matrix sets each condition against every other condition, in a pair-wise manner. Each pair of conditions is then examined, and a judgement is made as to whether - or to what extent - the pair can coexist, i.e. represent a consistent relationship. Note that there is no reference here to causality, but only to internal consistency.

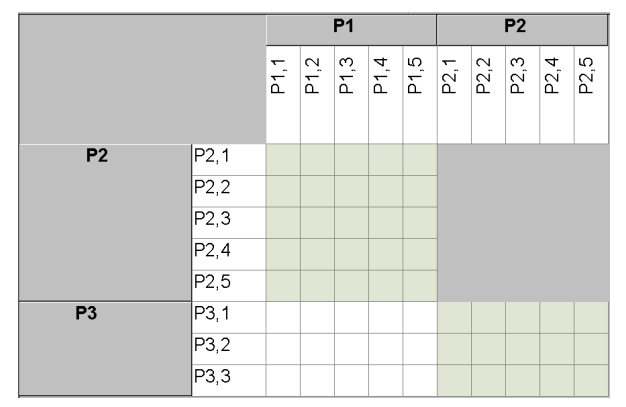

Figure 3: Cross-consistency matrix for the 3-parameter "Zwicky box".

Fields containing hundreds of thousands of configurations can typically be reduced by 90 or even 99 percent by making judgements about pairs that do not coexist. This reduction leaves us with a manageable number of configurations (i.e. solutions) to examine and work with.

The technique of using pair-wise consistency relationships between conditions, in order to weed out internally inconsistent configurations, is made possible by a principle of dimensionally reduction inherent in the morphological approach. While the number of configurations in a morphological field grows exponentially with each new parameter, the number of pair-wise relationships between conditions grows only as a quadratic polynomial. Naturally, practical limits may be reached even with quadratic growth. The point, however, is that a morphological field involving as many as 100000 formal configurations requires no more than a few hundred pair-wise evaluations in order to create a solution space.

With computer support, an internally assessed morphological field becomes a flexible model, in which anything can be 'input' and anything 'output.' This means that if a variable condition is chosen as an 'input,' then all conditions consistent to the input are highlighted as 'outputs' so that a map of consistent conditions is visible. Figure 5 is an example of a 'map' of consistent conditions. Such a morphological model or field can be turned into a laboratory with which one can designate single or multiple drivers, in order to examine outputs or solution alternatives.

Because of the complexity of the process, and the many thousands of potential configurations mapped out in even relatively small morphological fields, MA is difficult to employ without computer support. For this reason, the Swedish Defence Research Agency (FOI)

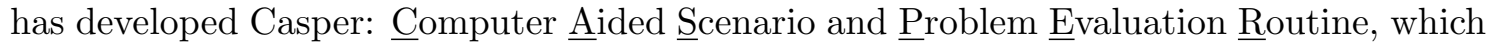
supports the entire MA-process. Casper is a proprietary software package developed and owned by the Swedish Defence Research Agency. 
MA goes through cycles of analysis and synthesis in a number of iterative steps. The iterative steps are (Ritchey \& Stenström, 2002):

Analysis phase: Define the problem complex in terms of variables and variable conditions.

Step 1: Identify the dimensions, parameters or variables, which best define the essential nature of the problem complex or scenario. One should work with no more than 6-7 variables at a time.

Step 2: For each variable, define a range of relevant, discrete values or conditions, which the variable can express. The variable and variable-condition matrix is the morphological field, an $n$-dimensional coordinate system that implicitly contains an outcome space for the problem complex thus defined.

Synthesis phase: Link variables and synthesise an outcome space.

Step 3: Assess the internal consistency of all pairs of variable conditions, weeding out all inconsistent or contradictory pairs. It is usually at this point that one begins to understand what the variables and variable conditions actually represent, that they are often poorly defined and must be adjusted. Steps 1 and 2 may now be reviewed and one may begin to iterate between steps 1, 2 and 3 until step 3 begins to work smoothly.

Step 4: Synthesise an internally consistent outcome space. (Casper does this by running through all of the possible formal solutions in the morphological field and "reducing" the field by restricting all outcomes containing internal contradictions. The surviving configurations represent the solution space of the given problem complex.

Step 5: Iterate the process if necessary. Scrutinise the solution space and return to steps 1, 2 and 3 in order to adjust variables, alternatives and consistency measures. Run steps 4 and 5 again. At this point, one has created a non-quantified "if-then" laboratory within which one can define drivers, assume certain conditions, and find the range of associated solutions.

The strength of morphological analysis lies in its focus on problem formulation, parameterisation and the establishment of an internal structure - all achieved through iterative cycles of analysis and synthesis, in a systematic and "traceable" manner. However, MA is weak in treating hierarchical structure, and does not establish causal relations between its constructs. For this, other modelling methods are required.

\section{Bayesian Networks}

\subsection{Causal Networks and $d$-separation}

One way to establish causal relationships and hierarchical structure between variables is to create a causal network of the problem complex. A causal network consists of nodes (variables) and arcs (directed links) between them, and mathematically it is called a directed graph. The relations between variables in a causal graph are explained by means of family relations: a link from $A$ to $B$ means that $B$ is a child of $A$ and $A$ is a parent of $B$ (Jensen, 2001). 
As with the MA technique, variables represent a set of conditions or states. In the real world, a variable is in exactly one of its states; however, the identity of the state may be unknown to us (Jensen, 2001). The purpose of the causal network is to understand how a change of states in one variable affects the certainty of states in other variables.

$d$-Separation is a property that describes the flow of information in a causal network, given that the state of a variable or set of variables is known. The nodes $X$ and $Y$ are $d$ separated if the set $Z$ is observed and because of that observation, no information can flow between $X$ and $Y$. $d$-Separation is reflected in the concept of conditional independence. The variables $A$ and $C$ are independent given the variable $B$ if $P\left(a_{i} \mid b_{j}\right)=P\left(a_{i} \mid b_{j}, c_{k}\right)$ for all $i, j, k$ (Jensen, 2001).

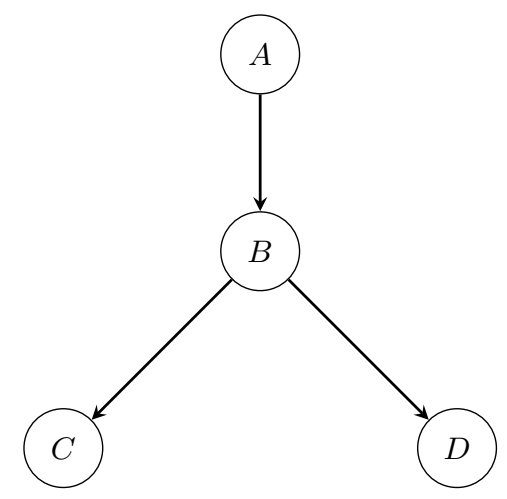

Figure 4: Simple Bayesian network

\subsection{Definition of Bayesian Networks}

Let $U=\left(X_{1}, \ldots, X_{n}\right)$ be a set of variables that describes a problem complex. Then a Bayesian network is a causal network representing the joint probability table $P(U)$ so that

$$
P(U)=\prod_{i} P\left(X_{i} \mid \operatorname{parents}\left(X_{i}\right)\right),
$$

where parents $\left(X_{i}\right)$ is the set of parents of $X_{i}$. This is the chain rule for a BN (Jensen, 2001). This concise representation of the joint probability $P(U)$ is made possible because of the $d$-separation property of directed graphs. Jensen (2001) gives the following definition of a BN. A Bayesian network consists of the following:

- A set of variables and a set of directed edges between the variables.

- Each variable has a finite set of mutually exclusive states.

- The variables together with the directed edges form a directed acyclic graph (DAG)

- To each variable $A$ with parents $B_{1}, \ldots, B_{n}$ there is attached the potential table $P\left(A \mid B_{1}, \ldots, B_{n}\right)$.

The definition of a $\mathrm{BN}$ does not refer to causality. It is rather the d-separation properties induced by the causal networks that is required to prove the chain rule for BNs (Jensen, 
2001). This definition also describes the process of developing a $\mathrm{BN}$, if each line in the definition is treated as a step in the development process.

The 'potential table' mentioned in line 4 of the definition describes the strength of relations between variables. This is achieved by means of probability calculus and the practical implementation is a conditional probability table (CPT) for each variable in the BN. Every variable is defined by the states (or conditions) that it takes on. States may be anything from sequential intervals to descriptions such as yes or no. The states of a variable must be mutually exclusive and exhaustive.

An example of the CPT for variable $C$ in the BN in Figure 4 is illustrated in Table 1. Note that the columns sum to one.

\begin{tabular}{llll}
\hline & $b_{1}$ & $b_{2}$ & $b_{3}$ \\
\hline$c_{1}$ & 0.3 & 0.4 & 0.2 \\
$c_{2}$ & 0.7 & 0.6 & 0.8 \\
\hline
\end{tabular}

Table 1: An example of $P(C \mid B)$ for the variable $C$ in Figure 4.

There are several potential sources for the probabilities of a BN. They include empirical data, literature and expert knowledge. Since the BN makes use of conditional probabilities, Bayes' rule for the calculation of conditional probabilities may be used. Mathematically, Bayes' rule states that

$$
P(X=x \mid e)=\frac{P(e \mid X=x) P(X=x)}{P(e)}
$$

where $P(X=x \mid e)$ denotes the probability that the random variable $X$ has value $x$ given evidence $e$ (Jensen, 2001). The denominator on the right hand side of the equation is a normalising constant. Once the $\mathrm{BN}$ is constructed, it is used to estimate the values of query nodes, given the values of observed nodes (Murphy, 2002). This process is called inference and a BN can perform two inference tasks. One is to perform top-down reasoning where 'root' nodes are observed and we predict the effects (Murphy, 2002) (option (a) in Table 1).

The other way is to perform bottom-up reasoning where 'leaf' nodes are observed and we infer the causes (Murphy, 2002) (option (b) in Table 1). The second task is the more interesting one as it reasons in the 'opposite' direction of the constructed arcs in the network. Figures 10-12 provide inference examples of a Bayesian network.

From a knowledge engineering perspective, a graphical model serves as a communication tool to capture knowledge of the domain. The construction of the graphical model can serve as a facilitation method for interpersonal communication. Humans can communicate sensibly about causal relations and the model helps to focus their attention. Secondly, the graphical model can communicate to a computer the knowledge captured during the interpersonal model building exercise. The computer should be able to process the model and perform what-if analyses (Jensen, 2001).

To sum up, when constructing a BN model, the major modelling criteria that arise are: 


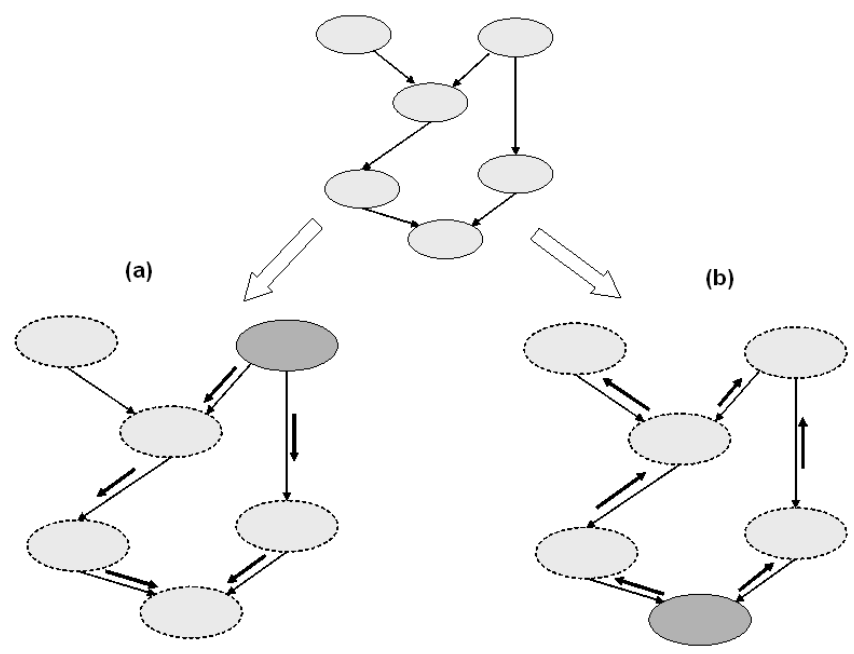

Figure 5: Illustration of two inference tasks in a $B N$

1. What are the variables and variable values?

2. What does the graphical (e.g. causal) structure look like - i.e. between which variables are there dependencies and what are their causal directions?

3. What are the strengths of these dependencies, as depicted in the graphical structure?

Although these issues are listed as sequential steps in the BN modelling process, in reality the process is far from this simple. Step 1 and 2 of developing a BN require considerable effort, but is more practicable (Druzdzel \& Van der Gaag, 2000). Step 3 of the process is considered the harder task and more time-consuming. Data sources available are not encoded in probabilities and many techniques exist to do that, depending on the data format. For example, the EM (Expectation-Maximisation) algorithm (Stuart \& Norvig, 2003) may be used to estimate the probabilities of a BN if empirical data are available. Many knowledge engineering techniques exist to elicit and translate expert knowledge into probabilities (Druzdzel \& Van der Gaag, 2000).

It is often the case of strategic decision support models, which are often extremely complex, that empirical data do not exist and the structure of the causal network is not intuitive to elicit. These features tend to leave expert groups with a sense of uneasiness in how to engage in an involved modelling process.

For this reason, we have found it advantageous to break up the modeling process into two conceptually distinct phases:

Phase 1: Dimension the problem by identifying relevant variables and variables values. Create a linked parameter space based on internal consistency assessment between all pairs of variable values. Although these internal relationships do not explicitly indicate a hierarchical or causal structure, they nevertheless give an indication of which variables significantly influence one another. This phase should be carried out without any reference to directed causality or hierarchy, thus allowing the working group to concentrate on one main task. This task can be facilitated with morphological analysis. 
Phase 2: On the basis of phase 1, create a directed, acyclic (causal) structure between the variables, and assess the strengths of the dependencies between the values of the dependent variables. This phase will result in a Bayesian network, which can be used as a "what-if" inference model.

\section{Case study}

As an example, we present a relatively small, prototype inference model, developed as proof-of-concept, for the Swedish Rescue Services Agency (SRSA). The case study involved developing a decision support model for assessing the environmental impact of different fire-fighting methods under different conditions.

The three pillars of rescue service operations concern protecting 1) human life, 2) property and 3) the environment - usually in that order. Thus, when rescue services are called upon to deal with fires, they must make a number of decisions on what method or methods they should employ. If lives are threatened, then any and all methods will be applied in order to save lives. However, when saving lives is not at stake, rescue services are required to take into account the environmental consequences of different fire fighting methods.

For instance, consider a container fire involving poisonous substances (e.g. heavy metals), located near a municipal water reserve or other ecologically sensitive area. Under such circumstances, fire fighters should avoid methods which would, for instance, result in uncontrolled spill water contaminating the environment.

For this reason, the Swedish Rescue Services Agency (SRSA) commissioned FOI (the Swedish Defence Research Agency in Stockholm) to develop a prototype decision support model in order to aid rescue service personnel in choosing appropriate fire fighting methods under different circumstances. The model was primarily to be utilized for planning, education and training, but might also be employed as an operational decision support system.

\subsection{Phase 1: Developing a morphological analysis model}

The model was developed during a series of workshops, each with the participation of 6-8 fire chiefs and fire engineers from different Swedish municipalities, and subject specialists from SRSA and FOI. The modelling process, as described in $\S 2$, was facilitated by the authors. The first step involved identifying and defining the problem area's most important variables. Seven variables were chosen for the prototype model:

\section{Situational variables (predictive inputs/diagnostic outputs):}

Type of event - i.e. what is the context of the fire?

Type of substance - i.e. what is burning?

Geographical situation - i.e. on what surface or by what medium can substances be spread?

Threatened recipient - i.e. what sensitive ecological areas or objects are in proximity?

Decision variables (throughput):

Fire-fighting methods available to the fire fighters. 
Consequence variables (predictive outputs/diagnostic inputs):

Likelihood of substance spreading to recipient, as the result of fighting the fire.

Environmental consequences of substance spreading.

These prototype variables and their subsequent values are shown in the morphological field below (Figure 5).

\begin{tabular}{|c|c|c|c|c|c|c|}
\hline $\begin{array}{l}\text { Type of } \\
\text { substance }\end{array}$ & Type of event & $\begin{array}{l}\text { Geological } \\
\text { situation }\end{array}$ & \begin{tabular}{|l} 
Type of \\
threatened \\
recipient
\end{tabular} & $\begin{array}{l}\text { Method } \\
\text { (of fire fighting) }\end{array}$ & $\begin{array}{l}\text { Likelihood of } \\
\text { substance } \\
\text { spreading }\end{array}$ & $\begin{array}{l}\text { Environmental } \\
\text { consequences }\end{array}$ \\
\hline Electrical waste & Container & Clay and rock & Surface water & Dry method & High & Irreversible \\
\hline Rubber & Industrial & $\begin{array}{l}\text { Stony pine seed } \\
\text { ground }\end{array}$ & Ground water & $\begin{array}{l}\text { Foam or water } \\
\text { which can be } \\
\text { controlled }\end{array}$ & Moderate & Long-term \\
\hline Plastic & Recycling depot & Gravel & $\begin{array}{l}\text { "Natura 2000" } \\
\text { (Protected areas) }\end{array}$ & Water, no control & Low & Short-term \\
\hline Petroleum & Residential & Frozen ground & Sewerage plant & Foam, no control & & $\begin{array}{l}\text { No significant } \\
\text { consequences }\end{array}$ \\
\hline $\begin{array}{l}\text { Radioactive } \\
\text { substances }\end{array}$ & Transport/road & Storm water & Forest & Do nothing & & \\
\hline $\begin{array}{l}\text { Other } \\
\text { environmentally } \\
\text { dangerous }\end{array}$ & Bush & $\begin{array}{l}\text { Slag water } \\
\text { (populated area) }\end{array}$ & Farmland & & & \\
\hline $\begin{array}{l}\text { Other diverse } \\
\text { products }\end{array}$ & Large building & Open waterway & Sea & & & \\
\hline & & Air & Urban area & & & \\
\hline
\end{tabular}

Figure 6: Morphological field for the environmental impact of different fire-fighting methods, with one hypothetical case displayed.

The next step is to perform a cross consistency assessment in order to determine which variables directly affect which other variables. A preliminary cross consistency assessment revealed the following:

1. The situational variables (columns 1-4 in Figure 6) were found to be largely hyper coherent, i.e. it would seem that all pair-wise value combinations are possible. Some combinations are undoubtedly more likely than others, but this is irrelevant from the point of view of what we are trying to model. We are not interested in the probability of different situations, but in the consequences of decisions made in the context of any possible situation. We therefore leave the cross consistencies between the situational variables open (i.e. everything is possible). This is seen by the empty cross consistency cells in Figure 6.

2. The primary constraints on the best fire-fighting methods are "type of substance" and "type of event."

3. The primary constraints on the likelihood of spreading are "fire-fighting methods" and "geographical situation."

4. Finally, the primary constraints on environmental consequences are "type of substance," "threatened recipient" and "likelihood of spreading."

5. All other "pare-wise" value combinations can be left open.

A subset of these relationships are shown in Figure 7, below. The areas marked "X" represent the dependent variables. The areas marked "S" represent interesting combinations 
of conditions and serves as a 'flagging' mechanism. In morphological analysis, consistence relationships infer no causal direction, but only establish dependency. Since we had already decided to map out these directed dependencies in a Bayesian network, we did not perform the actual cross-consistency assessment on the morphological field.

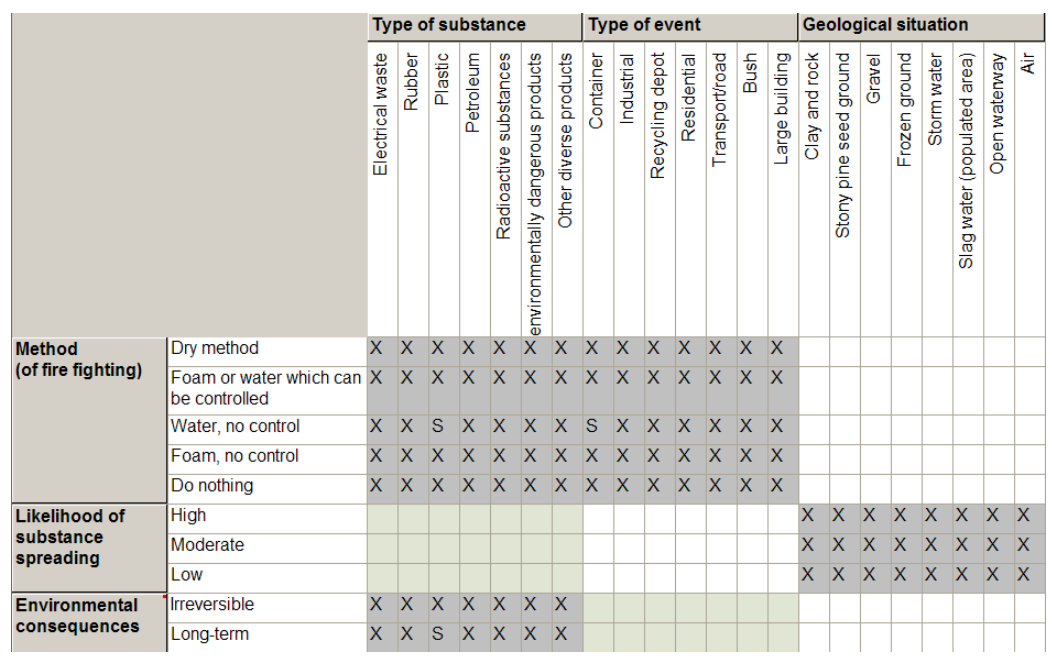

Figure 7: Cross-consistency matrix showing dependent variables (" $X$ " markings).

\subsection{Phase 2 - Developing a Bayesian network}

The preliminary cross consistency assessment revealed the variables which would display dependencies. The next step is to establish a causally directed structure, with multiple dependencies between certain variables. This step was undertaken with a group of fire engineers and fire chiefs during a two day workshop in Stockholm. This situation is represented by the causal network in Figure 8, which fulfils the second criterion for a Bayesian network model: "What does the graphical (e.g. causal) structure look like - i.e. between which variables are there dependencies and what are their causal directions?"

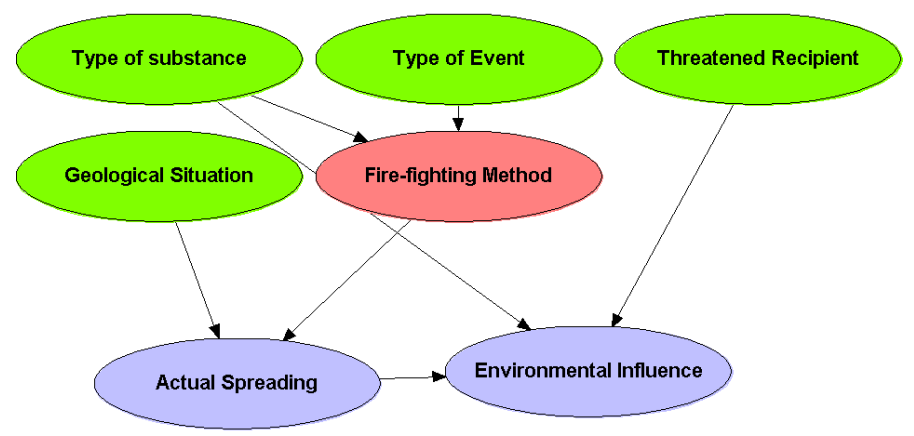

Figure 8: Causal network for the environmental impact of different fire-fighting methods.

The final step in producing the Bayesian network model is to establish the strengths of the dependencies between the relevant variables. The conditional probability tables were 
populated using the expert knowledge available at the workshop. This means that the quantitative information in the $\mathrm{BN}$ is based on expert judgement.

The next step in refining the $\mathrm{BN}$ is to encode the empirical data into probabilities and to use it (instead of expert knowledge) to populate the probability tables of the BN. This entails the task of estimating the probabilities from the data. Estimation methods include the maximum likehood procedure with complete data sets and the EM (ExpectationMaximisation) algorithm with incomplete data sets (Cowell et al., 1999). Figure 9 illustrates the CPTs of all the variables in the BN.

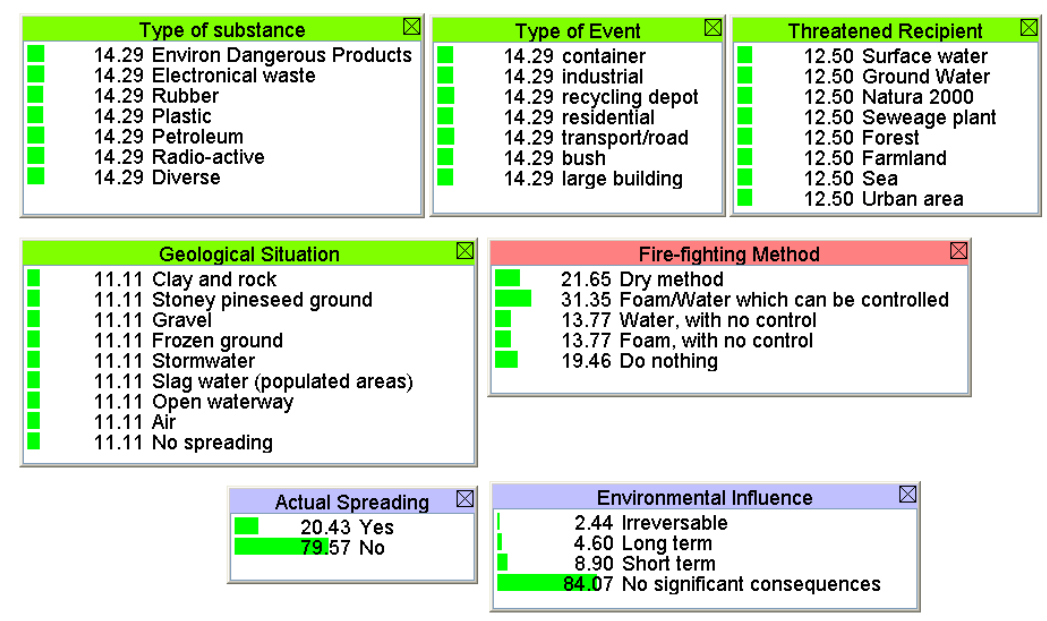

Figure 9: Quantified $B N$ for the environmental impact of different fire-fighting methods.

These CPTs are the results of a completed BN with quantitative information captured in the causal diagram (Figure 8) and quantitative information captured as probabilities in the CPTs. The links between variables remain as in the causal network in Figure 8 , but are omitted in Figures 9-12 to avoid visual clutter.

In both morphological and BN models, anything can be designated as "input," and anything as "output." As stated in $\S 3$, once the BN is constructed, it may be used to estimate values of query nodes (or variables), given the values of observed variables. In Figures 1012 the states with the number '100.00' represents the observed states of variables and all the other states represent the probabilistic values of those conditions given the observed states. The probabilities are normalised and multiplied by 100 so that the numbers in one CPT add up to 100 . The BN was constructed in the software package Hugin ${ }^{\circledR}$.

The resulting BN model may thus be used in a number of different modes. The first and most natural mode is synthetic and "predictive," i.e. given a set of circumstances and a selected fire-fighting method, what is the likelihood of substances spreading, leading to negative consequence for the environment. Figure 10 may be interpreted as follows: If

- 'Type of substance' = Plastic,

- 'Type of Event' = container,

- 'Geological Situation' = Frozen ground,

- 'Threatened Recipient' = Farmland, and 


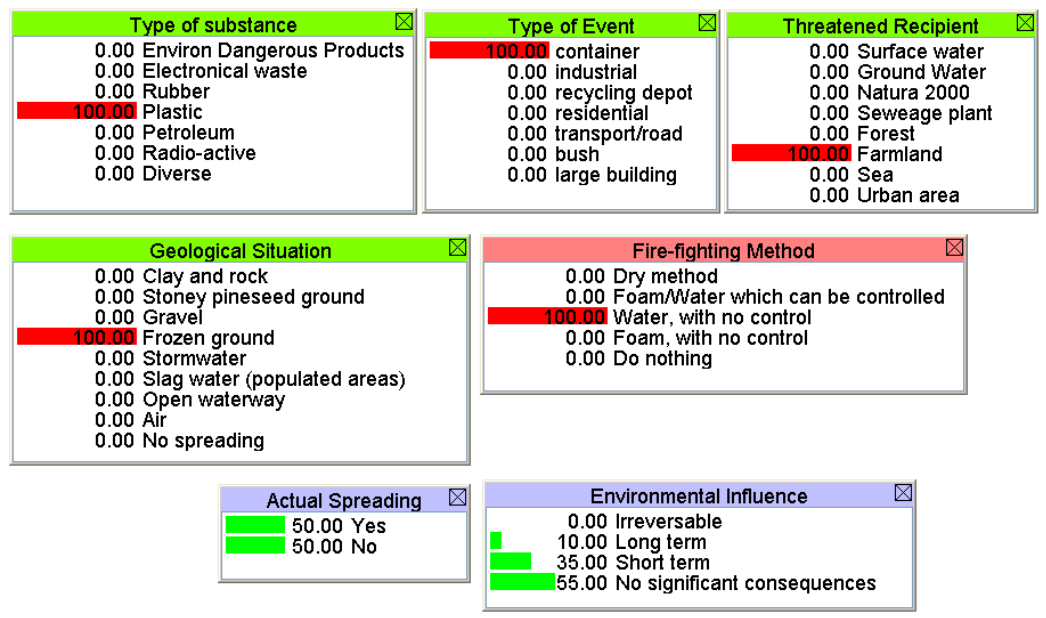

Figure 10: Example of Predictive mode. What if we use 'Water with no control' on a particular instance?

- 'Fire-fighting Method' = Water, with no control,

then there is a

- 0.55 probability for no significant environmental consequences,

- 0.35 probability for short term environmental influence,

- 0.1 probability for long term environmental influence, and

- 0 probability for irreversible environmental influence.

This may be seen from the CPT for the node "Environmental Influence" in Figure 10.

The second mode is synthetic and 'prescriptive,' i.e. given a set of circumstances, which fire-fighting methods are best for reducing substance spreading and resultant environmental damage (Figure 11). Finally, the third mode is analytic and 'diagnostic,' i.e. given a degree of environmental impact, what are the circumstances and fire-fighting methods that can lead to this (Figure 12). The interpretation of Figure 11 and Figure 12 is left to the reader.

These are only three examples. Any other combination of variables can be designated input, in order to check the output of the remaining variables, thus mixing predictive and diagnostic modes. This is especially valuable for education and training.

\section{Conclusions}

Both MA and BNs have proven to be appropriate techniques for treating complex, strategic problem spaces. MA is a method for defining, linking and evaluating these problem spaces. However, with MA it is difficult to represent hierarchical structure and the links between variables are not causally defined. Furthermore, the MA approach is non-quantified, which means that the strength of links between variables is not (quantitatively) defined. 


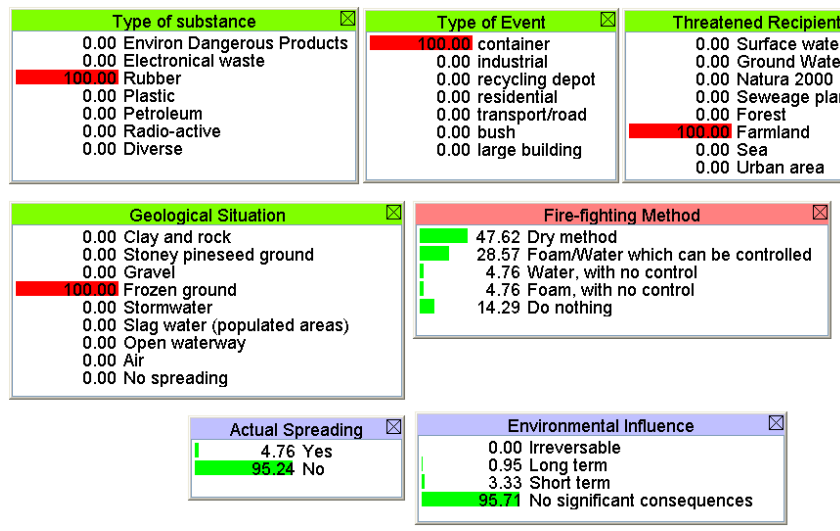

Figure 11: Prescriptive mode. What is the best fire-fighting method for a particular case?

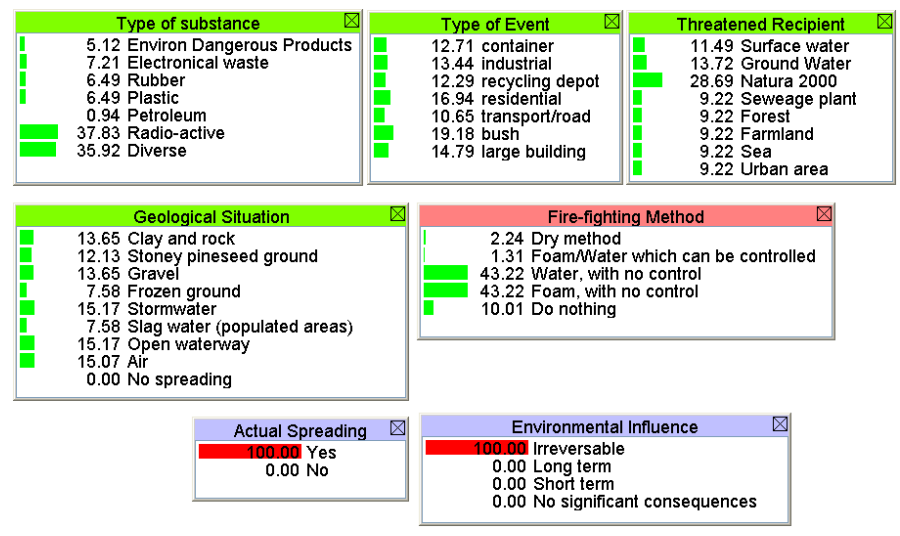

Figure 12: Example of Diagnostic mode: Under what circumstances can irreversible environmental damage be done?

BNs are graphical models which consist of a qualitative and quantitative part. The qualitative part is a cause-and-effect, or causal graph. The quantitative part depicts the strength of the causal relationships between variables. In terms of inference, a $\mathrm{BN}$ is much more descriptive, but also much more difficult to develop in the initial problem formulation phase of the modelling process.

We suggest a modelling process that makes use of both of these modelling techniques in two phases: Firstly the MA approach may be used to formulate the problem and to create an internal structure. This phase contributes to a better understanding of the problem space. In some instances this knowledge and understanding is not sufficient and there is a need for addressing hierarchical and/or causal structures. We then recommend the use of BNs in phase two of the modelling process. BNs introduce a causal structure and allow for quantification of the relationships between variables.

The model presented in the case study is only a 'proof-of-concept.' It is primarily intended to test a modelling method, not the subject area itself. However, the substantive decision support model for assessing the environmental impact of different fire-fighting methods under difference conditions is now in its next stage of development at the Swedish Rescue 
Services Agency. Also, a project involving decision support for phasing out military firing ranges has recently progressed from its morphological phase (Stenström \& Westin, 2004) to its Bayesian phase (not yet reported in English).

\section{References}

[1] Borsuk ME, Stow CA \& Reckhow KH, 2003, A Bayesian network of eutrophication models for synthesis, prediction, and uncertainty analysis, Ecological Modelling, 173, pp. 219-239.

[2] Cowell RG, Dawid, AP, Lauritzen, SL \& Spiegelhalter DJ, 1999, Probabilistic networks and expert systems, Springer-Verlag, New York (NY).

[3] Coyle RG \& McGlone GR, 1995, Projection Scenarios for South-east Asia and the South-west Pacific, Futures, 27(1), pp. 65-79.

[4] Coyle RG \& Yong YC, 1996, A scenario projection for the South China Sea, Futures, 28(3), pp. 269-283.

[5] Doty D \& Glick W, 1994, Typologies as a unique form of theory building, Academy of Management Review, 19, pp. 230-251.

[6] Druzdzel MJ \& van der GaAg LC, 2000, Building probabilistic networks: Where do the numbers come from?, IEEE Transactions on Knowledge and Data Engineering, 12(4), pp. 481-486.

[7] ERIKSSOn T \& Ritchey T, 2002, Scenario development and force requirements using morphological analysis, Paper presented at Winchester International OR Conference, Winchester, [Online], [Cited November 12th, 2007], Available at: http://www.swemorph.com/downloads.html

[8] Jensen FV, 2001, Bayesian networks and decision graphs, Springer-Verlag, New York (NY).

[9] Korb KB \& Nicholson AE, 2004, Bayesian artificial intelligence, Chapman \& Hall/CRC, Boca Raton (FL).

[10] Murphy KP, 2002, Dynamic Bayesian networks: Representation, inference and leaning, PhD dissertation, University of California, Berkeley.

[11] RHYNE R, 1981, Whole-pattern futures projection, using field anomaly relaxation, Technological Forecasting and Social Change, 19, pp. 331-360.

[12] Rhyne R, 1995a, Field anomaly relaxation — The arts of usage, Futures, 27(6), pp. 657-674.

[13] Rhyne R, 1995b, Evaluating alternative Indonesian Sea-sovereignty systems, Paper presented at Informs: Institute for Operations Research and the Management Sciences Conference, New Orleans.

[14] Ritchey T, 1997, Scenario development and risk management using morphological field analysis, Proceedings of the 5th European Conference on Information Systems (Cork: Cork Publishing Company) 3, pp. 1053-1059, [Online], [Cited November 12th, 2007], Available at: http://www. swemorph.com/downloads.html

[15] Ritchey T, 1998, Fritz Zwicky, 'Morphologie' and policy analysis, Paper presented at the 16th Euro Conference on Operational Analysis, Brussels.

[16] Ritchey T, 2002, Modelling complex socio-technical systems using morphological analysis, Adapted from an address to the Swedish Parliamentary IT Commission, Stockholm, December 2002, [Online], [Cited November 12th, 2007], Available at: http://www.swemorph.com/downloads.html 
[17] Ritchey T, 2003, Nuclear facilities and sabotage: Using morphological analysis as a scenario and strategy development laboratory, Adapted from astudy for the Swedish Nuclear Power Inspectorate, and presented to the 44th Annual Meeting of the Institute of Nuclear Materials Management, Phoenix, Arizona, [Online], [Cited November 12th 2007], Available at: http://www. swemorph.com/downloads.html

[18] Ritchey T \& Stenström M, 2002, Using morphological analysis to evaluate preparedness for accidents involving hazardous materials, Study for the Swedish Rescue Services Board, presented at the 4th International Conference for Local Authorities, Shanghai, [Online], [Cited November 12th, 2007], Available at: http://www.swemorph.com/downloads.html

[19] Stenström M \& Westrin P, 2004, Living with UXO - Using morphological analysis for decision support in phasing out military firing ranges, Summary of Report to the Swedish Armed Forces UXO Program, Stockholm, [Online], [Cited November 12th, 2007], Available at: http://www. swemorph.com/downloads.html

[20] Stuart R \& Norvig P, 2003, Artificial intelligence - A modern approach, Pearson Education Inc., Englewood Cliffs (NJ).

[21] ZwiCKy F, 1969, Discovery, invention, research - Through the morphological approach, The Macmillian Company, Toronto.

[22] Zwicky F \& Wilson A (EDs.), 1967, New methods of thought and procedure: Contributions to the symposium on methodologies, Springer, Berlin, [Online], [Cited November 12th, 2007], Available at: http://www.swemorph.com/zwicky.html 
